\title{
Cross-Validation of the Taiwan Version of the Moorehead-Ardelt Quality of Life Questionnaire II with WHOQOL and SF-36
}

\author{
Chi-Yang Chang • Chih-Kun Huang • Yu-Yin Chang • \\ Chi-Ming Tai $\cdot$ Jaw-Town Lin $\cdot$ Jung-Der Wang
}

Received: 3 December 2008 / Accepted: 10 February 2009/Published online: 3 March 2009

(C) The Author(s) 2009. This article is published with open access at Springerlink.com

\begin{abstract}
Background Obesity has become a major worldwide public health issue. There is a need for tools to measure patientreported outcomes. The Moorehead-Ardelt Quality of Life Questionnaire II (MA II) contains six items. The objective of this study was to translate the MA II into Chinese and validate it in patients with morbid obesity.

Methods The MA II was translated into Chinese and backtranslated into English by two language specialists to create the Taiwan version, which was validated by correlations with two other generic questionnaires of health-related quality of life (HRQOL), Medical Outcomes Study 36-Item Short-Form Health Survey (SF-36), and World Health Organization Quality of Life (WHOQOL)-BREF Taiwan version. The convergent validity was accomplished by a
\end{abstract}

C.-Y. Chang $\cdot$ C.-M. Tai $\cdot$ J.-T. Lin

Department of Internal Medicine, E-Da Hospital,

I-Shou University,

Kaohsiung, Taiwan

C.-K. Huang

Department of Surgery, E-Da Hospital, I-Shou University,

Kaohsiung, Taiwan

C.-Y. Chang $\cdot$ J.-T. Lin $\cdot$ J.-D. Wang $(\triangle)$

Department of Internal Medicine,

National Taiwan University Hospital,

Taipei, Taiwan

e-mail: jdwang@ntu.edu.tw

J.-D. Wang

Department of Environmental and Occupational Medicine,

National Taiwan University Hospital,

Taipei, Taiwan

C.-Y. Chang $\cdot$ Y.-Y. Chang $\cdot$ J.-D. Wang

Institute of Occupation Medicine and Industrial Hygiene,

College of Public Health, National Taiwan University,

Taipei, Taiwan series of Spearman rank correlations. Reliability of the MA II Taiwan version was determined by internal consistency obtained by Cronbach's alpha coefficient and test-retest reliability obtained by intraclass correlation coefficient.

Results One hundred subjects with morbid obesity were enrolled to test the MA II Taiwan version convergent validity and internal consistency. Test-retest studies ( 2 weeks apart) were applied to 30 morbidly obese patients. Satisfactory internal consistency was demonstrated by a Cronbach's alpha coefficient of 0.79 . Good test-retest reliability was shown by intraclass correlations ranging from 0.73 to 0.91 . The total sum of MA II scores was significantly correlated with all four domains of the WHOQOL-BREF and two major components of SF-36 (all correlations, $p<0.01$; range, $0.44-0.64)$. All six MA II items showed significant correlations with each other $(r=0.34-0.69, p<0.01)$, and the total sum of MA II scores was negatively correlated with body mass index $(r=-0.31, p<0.01)$, indicating a onedimensional questionnaire of HRQOL.

Conclusions The MA II Taiwan version is an obesity-specific questionnaire for QOL evaluation with satisfactory reliability and validity. It has the advantages of extensive evaluation for HRQOL, cross-cultural application, rapid completion, high response rates, and an advanced scoring system.

Keywords Morbid obesity · Quality of life · Validation . WHOQOL-BREF.SF-36

\section{Introduction}

The prevalence of obesity has increased markedly in the past 20 years, becoming a major public health issue not only in the western countries but also in Asia [1-5]. Many obesity-related comorbidities have been documented $[6,7]$. 
These comorbidities consume considerable medical resources worldwide. Individual quality of life is also significantly influenced by obesity. In general, increases in body mass index (BMI) are correlated with poorer health-related quality of life (HRQL) [8]. Many instruments are currently available to measure the HRQL of subjects with obesity, including the Medical Outcomes Study 36-Item Short-Form Health Survey (SF-36) [9-12], the World Health Organization Quality of Life (WHOQOL) instruments [8], and the Impact of Weight on Quality of Life (IWQoL) questionnaire [13, 14]. Of the many methods for treating obesity that have been introduced, bariatric surgery and behavioral and pharmacologic methods are reported to be effective based on short-term and/or longterm weight loss [15-17], decrease in the incidence of comorbidities, and improvement in patients' HRQL [9, 1820]. To corroborate this evidence, it is crucial to have a sensitive psychometric tool for detection of obesity-related impairment of quality of life.

The Moorehead-Ardelt Quality of Life Questionnaire, part of the Bariatric Analysis and Reporting Outcome System (BAROS) [21], is a condition-specific instrument for use with obesity that can be used independently. BAROS has been utilized in many countries [11, 22-24] and has become one of the standard outcome evaluations for bariatric surgery. The original Moorehead-Ardelt Quality of Life Questionnaire was created to measure postoperation outcome. This questionnaire was improved in 2003 in a second edition Moorehead-Ardelt Quality of Life Questionnaire II (MA II) based on suggestions from users [25]. MA II contains six items (Q1-Q6) and can be applied for both pre-intervention and postintervention assessments [26].

The WHOQOL group was initiated by the World Health Organization in 1991 [27, 28]. The WHOQOL is a generic QOL instrument with two unique features. It assesses physical, psychological, social, and environment domains comprehensively. It is also a cross-cultural questionnaire developed for use across different patient groups located in different countries [29]. The WHOQOL group later simplified the standard questionnaire to a short form called WHOQOLBREF [30]. This simplified questionnaire is a sensitive tool for evaluation of HRQOL in patients with various diseases, including morbid obesity [8,31-34]. The aims of the present study were to translate the MA II into Chinese and then crossvalidate it with the Taiwan version of WHOQOL-BREF and SF-36 among subjects presenting at an obesity clinic.

\section{Methods}

\section{Patients}

This study was approved by the Institutional Ethics Committee of E-Da Hospital, I-Shou University. All patients with morbid obesity who came to the Bariatric Center before or after surgery during January 2007-July 2008 were invited into this study. Each patient signed an informed consent form prior to the interview. The surgery has been regularly performed in this hospital and the majority received laparoscopic Roux-en-Y gastric bypass procedure. We adopted the recommendation of the AsiaPacific consensus [5], which stipulates that to be eligible for bariatric surgery, patients must be between age 18 and 65 years and their BMI must fall between 32 and $40 \mathrm{~kg} / \mathrm{m}^{2}$ with obesity related comorbidities, or their BMI must be more than $40 \mathrm{~kg} / \mathrm{m}^{2}$.

Translation of the Moorehead-Ardelt Quality of Life Questionnaire II

We obtained the permission of professor Moorehead to translate and validate the MA II into a Taiwanese version. The MA II was translated into Chinese and back-translated into English, respectively, by two specialists familiar with these two languages to create the Taiwan version. Five patients with morbid obesity were invited to check the wording and meanings of the original MA II Taiwan version. Finally, the MA II Taiwan version was created after careful discussion in the expert focus group, which was composed of a dietician, a nurse, and physicians with five different specialties (namely, psychiatry, obstetricsgynecology, surgery, internal medicine, and urology).

\section{Validation of the Moorehead-Ardelt Quality of Life Questionnaire II}

The MA II Taiwan version is the same as the original questionnaire, which includes six items (Q1: general selfesteem, Q2: physical activity, Q3: social contacts, Q4: satisfaction concerning work, Q5: pleasure related to sexuality, Q6: focus on eating behavior) and specific color-illustrated graphic symbols to help patients to understand the items cross-culturally. With this version, the score of each item is equally weighted and uses a tenpoint Likert scale. The reliability of the MA II Taiwan version was determined by internal consistency obtained by Cronbach's alpha coefficient and the test-retest reliability was obtained using an intraclass correlation coefficient (ICC).

The MA II Taiwan version was validated by its concordance with two other generic health-related quality of life questionnaires, the Taiwan versions of the SF-36 and the WHOQOL-BREF. The convergent validity was tested by a series of Spearman rank correlations among the three questionnaires. The Taiwan version of the WHOQOL-BREF contains four domains (physical, psychological, social, and environment), including the 26 original items of the 
WHOQOL-BREF, plus two culture-specific questions as national items for Taiwan. One item addressing "respect from others" was categorized into the social domain and another corresponded to "eating what one likes to eat" and was included in the environment domain. The method of application, the scoring procedures, and reference time point (during the last 2 weeks) were the same as for the original WHOQOL-BREF [28]. The SF-36 Taiwan version is the first version of SF-36, which contains eight scales (physical functioning, physical role, bodily pain, general health, vitality, social function, emotional role, and mental health). The method of application, the scoring procedures, and the reference time point (during the last 4 weeks) were the same as for the original SF-36 [35].

\section{Statistical Analysis}

We first conducted a descriptive analysis and listed the frequencies of the patients' demographic characteristics. Internal consistency was indicated by Cronbach's alpha coefficient, which was considered useful at a level 0.70 . The test-retest reliability was determined by ICC. An ICC value of $0.51-0.70$ indicated acceptable agreement, an ICC value of $0.71-0.90$ meant good agreement, and an ICC value greater than 0.90 indicated almost excellent agreement. Different domains of the WHOQOL-BREF and different scales of the SF-36 were subsequently summarized. The total scores for MA II were obtained by the sum of scores from each item. The convergent validity was accomplished by a series of Spearman rank correlations between MA II and the aforementioned two generic health related QOL questionnaires, WHOQOLBREF and SF-36. The test instruments were considered significant at $p<0.01$. Multiple linear regression models were constructed by using the total sum of MA II scores as the dependent variables, while BMI, age, sex, years of education, employment, and marital status were included as the independent predictive variables. A forward stepwise strategy was applied to select significant independent variables with $p<0.05$ as the inclusion criterion. All data were collected and analyzed using of SPSS Version 12.0.

\section{Results}

Demographic Characteristics

One hundred obese patients seen at the E-Da Hospital Bariatric Center between January 2007 and July 2008 either before or after surgery were enrolled in this study. Patient demographics and clinical characteristics are summarized in Table 1. The mean patient age was 31.0 years and their mean BMI was $35.6 \mathrm{~kg} / \mathrm{m}^{2}$. The majority $(69 \%)$ of obese subjects were women. The proportion of comorbidity with diabetes mellitus was $13 \%$. No subjects had suffered from stroke, myocardial infarction, or end stage renal disease which would significantly impair HRQOL.

\section{Reliability of MA II Taiwan Version}

Satisfactory internal consistency was demonstrated by a Cronbach's alpha coefficient of 0.79 . Good agreement on test-retest reliability was based on high intraclass correlations: general self-esteem, 0.89 (range, 0.79-0.95); physical activity, 0.73 (range, 0.51-0.86); social contacts, 0.77 (range, 0.58-0.89); satisfaction concerning work, 0.89 (range, 0.77-0.94); pleasure related to sexuality, 0.91 (range, 0.82-0.96); and focus on eating behavior, 0.81 (range, 0.65-0.91).

\section{Validity of MA II Taiwan Version}

The total sums of MA II scores were significantly correlated with all four domains of the WHOQOL-BREF and two major components of SF-36 (all correlations, $p<0.01)$. The WHOQOL-BREF domains were physical $(r=0.48)$, psychological $(r=0.64)$, social $(r=0.58)$, and environment $(r=0.44)$. The SF-36 components were physical health $(r=0.49)$ and mental health $(r=0.58)$. The MA II scores were also correlated with all eight scales of the SF-36 (all correlations, $p<0.01)$ : physical functioning $(r=0.48)$, physical role $(r=0.34)$, bodily pain $(r=0.29)$, general health $(r=0.53)$, vitality $(r=0.49)$, social function $(r=0.58)$, emotional role $(r=0.38)$, and mental health $(r=0.45)$. The

Table 1 Demographic characteristics of patients with obesity enrolled for validation of MA II, Taiwan version

\begin{tabular}{llll}
\hline & Preoperation $(n=50)$ & Postoperation $(n=50)$ & Overall $(n=100)$ \\
\hline Age (mean $\pm \mathrm{SD}$, range) & $31.6 \pm 10.4(18-59)$ & $30.3 \pm 9.2(19-53)$ & $31.0 \pm 9.8$ \\
Sex $(\%$ female) & 66.0 & 72.0 & 69.0 \\
Diabetes mellitus $(\%)$ & $14.0 \%$ & $12.0 \%$ & $13.0 \%$ \\
Weight $(\mathrm{kg}$; mean $\pm \mathrm{SD}$, range) & $109.6 \pm 26.8(80.0-201.0)$ & $90.9 \pm 23.6(57.8-160.6)$ & $99.8 \pm 26.7$ \\
Height $(\mathrm{cm}$; mean $\pm \mathrm{SD}$, range) & $167.8 \pm 10.6(150.3-182.8)$ & $166.5 \pm 8.3(150.4-180.8)$ & $167.1 \pm 9.4$ \\
BMI $\left(\mathrm{kg} / \mathrm{m}^{2}\right.$; mean $\pm \mathrm{SD}$, range) & $38.9 \pm 9.2(31.5-69.5)$ & $32.6 \pm 6.9(22.3-50.0)$ & $35.6 \pm 8.7$ \\
\hline
\end{tabular}


Table 2 Correlation of MA II, WHOQOL-BREF, and SF-36 scores among preoperation and postoperation groups

\begin{tabular}{|c|c|c|c|c|c|c|c|c|c|c|c|c|c|}
\hline & \multirow[t]{2}{*}{ MA II } & \multicolumn{2}{|c|}{ WHOQOL } & \multicolumn{10}{|l|}{ SF-36 } \\
\hline & & Phy & Psy & Soc & Env & $\mathrm{PF}$ & PR & $\mathrm{BP}$ & GH & VT & $\mathrm{SF}$ & ER & $\mathrm{MH}$ \\
\hline Pre-OP $(n=50)$ & 0.15 & $11.7^{*}$ & $11.3 * *$ & $12.7 * *$ & $13.5^{* *}$ & 68.6 & 49.5 & $65.6^{*}$ & $39.6^{* *}$ & $52.0 *$ & $64.5^{* *}$ & 53.3 & $59.2 *$ \\
\hline Post-OP $(n=50)$ & 1.16 & $12.3 * *$ & $12.5^{* *}$ & $14.3^{* *}$ & $13.8 * *$ & $81.2 *$ & 83.3 & 72.9 & $67.4^{*}$ & $64.3 * *$ & $77.0^{* *}$ & 85.8 & $67.3 * *$ \\
\hline Overall $(n=100)$ & 0.65 & $12.0 * *$ & $11.9 * *$ & $13.5^{* *}$ & $13.6^{* *}$ & $77.4^{* *}$ & $64.8 * *$ & $68.6 * *$ & $52.7 * *$ & $57.5^{* *}$ & $70.0^{* *}$ & $67.0^{* *}$ & $61.9 * *$ \\
\hline
\end{tabular}

Phy physical, $P s y$ psychological, $S o c$ social, Env environment, $P F$ physical functioning, $P R$ physical role, $B P$ bodily pain, $G H$ general health, $V T$ vitality, $S F$ social function, $E R$ emotional role, $M H$ mental health, Pre-OP preoperation, Post-OP postoperation

${ }^{*} p<0.05,{ }^{*} p<0.01$ (Spearman rank correlation among the total sum of MA II scores, WHOQOL-BREF domain scores, and SF-36 scale scores)

results of Spearman rank correlations among the total sum of MA II scores, WHOQOL-BREF, and SF-36 were similar in both preoperation and postoperation groups (Table 2). Matrix of correlation coefficient showed that each individual item on the MA II Taiwan version had extensive correlations with the domain scores of WHOQOL-BREF and dimension scores of SF-36 (Table 3), indicating an acceptable convergent validity. The fifth item (Q5) focusing on pleasure related to sexuality had a more significant correlation with the social and psychological domains of the WHOQOL-BREF, but less so with the two components of SF-36. Similarly, the sixth item focusing on eating behavior was more significantly correlated with the environmental and social domains of WHOQOL-BREF.
As shown in Fig. 1, the total sum of MA II scores showed a significant negative correlation with BMI $(r=-0.31$, $p<0.01$ ). After adjusting for other risk factors, multiple linear regression models showed that only BMI and employment were independent variables affecting the total sum of MA II scores (Table 4).

\section{Discussion}

The most important finding from this study is the broad coverage of QOL concepts by the MA II questionnaire. With just six questions, it measures all four domains and two components included in the generic questionnaires

Table 3 Matrix of correlation coefficients among the WHOQOL-BREF, SF-36, and MA II scores

Spearman $r$

\begin{tabular}{|c|c|c|c|c|c|c|c|c|c|c|c|c|}
\hline & \multicolumn{2}{|c|}{ WHOQOL } & \multicolumn{4}{|l|}{ SF-36 } & \multicolumn{6}{|l|}{ MA II } \\
\hline & Phy & Psy & Soc & Env & $\mathrm{PH}$ & $\mathrm{MH}$ & Q1 & Q2 & Q3 & Q4 & Q5 & Q6 \\
\hline \multicolumn{13}{|c|}{ WHOQOL } \\
\hline Phy & 1.00 & $0.65^{*}$ & $0.62 *$ & $0.58^{*}$ & 0.07 & 0.16 & $0.53 *$ & $0.40 *$ & $0.43^{*}$ & $0.45^{*}$ & 0.25 & 0.18 \\
\hline Psy & & 1.00 & $0.63 *$ & $0.60^{*}$ & 0.25 & 0.31 & $0.60 *$ & $0.54 *$ & $0.64 *$ & $0.41^{*}$ & $0.33^{*}$ & $0.33 *$ \\
\hline Soc & & & 1.00 & $0.62 *$ & $0.42 *$ & $0.42 *$ & $0.50 *$ & $0.49 *$ & $0.58 *$ & $0.44^{*}$ & $0.39 *$ & $0.40 *$ \\
\hline Env & & & & 1.00 & 0.07 & 0.23 & $0.40 *$ & $0.31 *$ & $0.41 *$ & $0.42 *$ & $0.30 *$ & $0.41 *$ \\
\hline \multicolumn{13}{|l|}{ SF-36 } \\
\hline $\mathrm{PH}$ & & & & & 1.00 & $0.67 *$ & $0.42 *$ & $0.51 *$ & $0.37 *$ & $0.27 *$ & 0.21 & $0.28 *$ \\
\hline $\mathrm{MH}$ & & & & & & 1.00 & $0.57 *$ & $0.51 *$ & $0.55^{*}$ & $0.38^{*}$ & $0.27 *$ & $0.36^{*}$ \\
\hline \multicolumn{13}{|l|}{ MA II } \\
\hline Q1 & & & & & & & 1.00 & $0.69 *$ & $0.65^{*}$ & $0.60^{*}$ & $0.45^{*}$ & $0.35^{*}$ \\
\hline Q2 & & & & & & & & 1.00 & $0.62 *$ & $0.50^{*}$ & $0.50 *$ & $0.42 *$ \\
\hline Q3 & & & & & & & & & 1.00 & $0.42 *$ & $0.53 *$ & $0.37 *$ \\
\hline Q4 & & & & & & & & & & 1.00 & $0.38^{*}$ & $0.34^{*}$ \\
\hline Q5 & & & & & & & & & & & 1.00 & $0.42 *$ \\
\hline Q6 & & & & & & & & & & & & 1.00 \\
\hline
\end{tabular}

Phy physical, Psy psychological, Soc social, Env environment, $P H$ physical health, $M H$ mental health $* p<0.01$ 


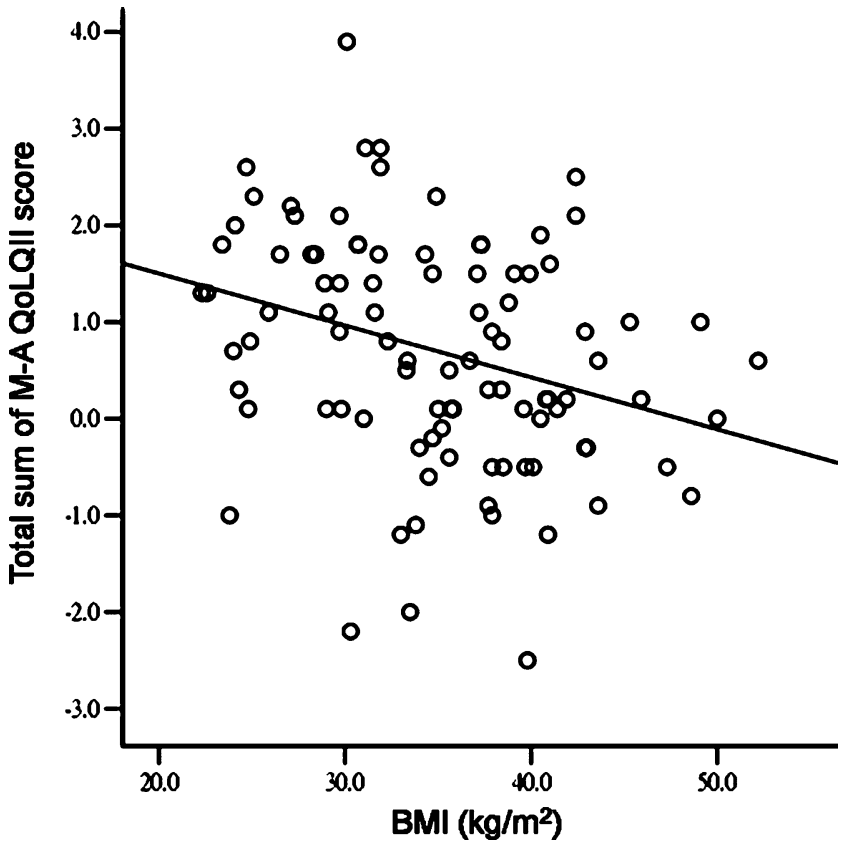

Fig. 1 The total sum of MA II scores showed a significant negative correlation with BMI $(r=-0.31, p<0.01)$

WHOQOL-BREF and SF-36, respectively, as shown in the correlation matrix presented in Table 3 . We also found that all six items of MA II were significantly correlated with each other $(r=0.34-0.69 ; p<0.01)$. With the additional evidence of significant correlation between the total sum of MA II and BMI level (Fig. 1), the present study corroborates the hypothesis that MA II may be a onedimensional questionnaire of HRQOL [26]. If we collect the postoperation group with a longer time after surgery, such as 1 year, or include the normal population without obesity, the change of BMIs value could possibly be more dramatic. Thus, future studies of responsiveness of MA II had better include subjects with a wider range of BMIs to corroborate the above prediction. The MA II scoring system has been changed so that each item was weighted equally under a ten-point Likert scale. Thus, the total sum of scores represented the HRQOL of obese patients and would be a suitable indicator for future cost-effective analyses focused on controlling obesity.

Although generic instruments, such as WHOQOL-BREF [8] or SF-36 [9-12], have been proven to be useful for measuring QOL in an obese population, these surveys have
26 and 36 items, respectively, and generally require a longer period of time for completion. For comparison, a review by Duval et al. [36] reported that nine other obesityspecific quality of life questionnaires contained between eight and 74 items. For example, the IWQoL questionnaire, which is probably the most frequently used obesity-specific QOL questionnaire with good reliability, validity, and responsiveness, has proven its clinical usefulness in many studies $[13,14]$. However, this survey includes 74 items and even the IWQoL-Lite has 31 items. On the other hand, the MA II has only six questions. In our experience, it could be completed within 1 or $2 \mathrm{~min}$ and the response rate was usually above $95 \%$ in clinical application. Moreover, each question of the MA II has a specific color-illustrated graphic symbol to assist patients in understanding the concepts cross-culturally. In this study, we applied the WHOQOL-BREF and SF-36 in our measurements; both of which have been demonstrated as excellent cross-cultural instruments for different patient groups and in different countries [29, 37-39]. Through cross-validation with the above two instruments, MA II has demonstrated its excellent cross-cultural potential.

MA II [26] was modified from the first edition of Moorehead-Ardelt Quality of Life Questionnaire [21]. The wording has been changed to be less suggestive so that it can be utilized with obese subjects either before or after operation. Moorhead et al. [26] validated the MA II by testing 110 obese patients before bariatric surgery. In our study, MA II data were collected from subjects with obesity both preoperation and postoperation. More than satisfactory reliabilities were obtained with a Cronbach's alpha coefficient of 0.79 and intraclass correlation coefficients between 0.73 and 0.91 . The present study also demonstrated good convergent validity between MA II and SF-36, as well as MA II and WHOQOL-BREF (Table 2). The MA II scores were correlated with all eight scales of the SF-36 (all correlations, $p<0.01)$. However, the American validation project of the MA II did not correspond to physical functioning scale of SF-36 [26]. The difference might be due to the different characteristics of study populations between our study and the American validation project. Since we included both preoperation and postoperation subjects, the ranges of the BMI and QOL values would be wider, which more likely showed a statistically significant correlation. Table 2 indicated that the MA II scores had no
Table 4 Multiple linear regression analysis of total sum of MA II scores in patients with morbid obesity

\begin{tabular}{lcccr}
\hline & Regression coefficient & Standard error & Partial $R$ square & $p$ value \\
\hline Constant & 1.97 & 0.67 & & \\
BMI $\left(\mathrm{kg} / \mathrm{m}^{2}\right)$ & -0.05 & 0.02 & 0.09 & $<0.01$ \\
Employment & 0.59 & 0.28 & 0.04 & 0.04 \\
\hline
\end{tabular}


correlation with the score of physical functioning scale in SF-36 for the preoperation individuals only, but showed a significant correlation after we included the postoperation subgroup. Good MA II construct validity was also validated by significant correlations with all domains of the WHOQOL-BREF $(p<0.01$; Table 2$)$. Therefore, the MA II was proven to be useful not only with obese patients after operation but also with patients before intervention.

In conclusion, the MA II Taiwan version is an obesityspecific questionnaire for QOL evaluation with satisfactory reliability and validity. It has the advantages of comprehensive coverage of four domains of QOL, cross-culture application, consists of only six items (time-saving) with an advanced scoring system, and is most likely unidimensional. Future demonstration of responsiveness may be useful for wider clinical application.

Acknowledgment This study is partially supported by a project from the National Science Council, Executive Yuan of Taiwan (962628-B-002-071-MY3). We thank Melodie K. Moorehead, Ph.D., ABPP to permit us to translate and validate the MA II into a Taiwanese version. Both the BAROS and MA II are copyright protected instruments and Lifetime Licensing Agreements are available at the website (http://www.drmoorehead.com/).

Disclosure None of the authors holds any significant financial interest in the product discussed that would represent a conflict of interest.

Open Access This article is distributed under the terms of the Creative Commons Attribution Noncommercial License which permits any noncommercial use, distribution, and reproduction in any medium, provided the original author(s) and source are credited.

\section{References}

1. Huang KC. Obesity and its related diseases in Taiwan. Obes Rev. 2008;9(Suppl 1):32-4.

2. Ogden CL, Carroll MD, Curtin LR, et al. Prevalence of overweight and obesity in the United States, 1999-2004. JAMA 2006;295:1549-55.

3. Prentice AM. The emerging epidemic of obesity in developing countries. Int J Epidemiol. 2006;35:93-9.

4. Sturm R. Increases in clinically severe obesity in the United States, 1986-2000. Arch Intern Med. 2003;163:2146-8.

5. Lee WJ, Wang W. Bariatric surgery: Asia-Pacific perspective. Obes Surg. 2005;15:751-7.

6. Bray GA. Medical consequences of obesity. J Clin Endocrinol Metab. 2004;89:2583-9.

7. Li Z, Bowerman S, Heber D. Health ramifications of the obesity epidemic. Surg Clin North Am. 2005;85:681-701.

8. Chang CY, Hung CK, Chang YY, et al. Health-related quality of life in adult patients with morbid obesity coming for bariatric surgery. Obes Surg. 2009; in press.

9. Livingston EH, Fink AS. Quality of life: cost and future of bariatric surgery. Arch Surg. 2003;138:383-8.

10. Choban PS, Onyejekwe J, Burge JC, et al. A health status assessment of the impact of weight loss following Roux-en-Y gastric bypass for clinically severe obesity. J Am Coll Surg. 1999; 188:491-7.

11. Nguyen NT, Goldman C, Rosenquist CJ, et al. Laparoscopic versus open gastric bypass: a randomized study of outcomes, quality of life, and costs. Ann Surg. 2001;234:279-89, discussion 289-91.

12. Dymek MP, Le Grange D, Neven K, et al. Quality of life after gastric bypass surgery: a cross-sectional study. Obes Res. 2002;10:1135-42.

13. Kolotkin RL, Head S, Hamilton M, et al. Assessing impact of weight on quality of life. Obes Res. 1995;3:49-56.

14. Kolotkin RL, Crosby RD. Psychometric evaluation of the impact of weight on quality of life-lite questionnaire (IWQOL-lite) in a community sample. Qual Life Res. 2002;11:157-71.

15. Maggard MA, Shugarman LR, Suttorp M, et al. Meta-analysis: surgical treatment of obesity. Ann Intern Med. 2005;142:547-59.

16. Delinsky SS, Latner JD, Wilson GT. Binge eating and weight loss in a self-help behavior modification program. Obesity 2006;14:1244-9.

17. Davis MM, Slish K, Chao C, et al. National trends in bariatric surgery, 1996-2002. Arch Surg. 2006;141:71-4, discussion 75.

18. Engel SG, Crosby RD, Kolotkin RL, et al. Impact of weight loss and regain on quality of life: mirror image or differential effect? Obes Res. 2003;11:1207-13.

19. Glinski J, Wetzler S, Goodman E. The psychology of gastric bypass surgery. Obes Surg. 2001;11:581-8.

20. Christou NV, Sampalis JS, Liberman M, et al. Surgery decreases long-term mortality, morbidity, and health care use in morbidly obese patients. Ann Surg. 2004;240:416-23, discussion 423-4.

21. Oria HE, Moorehead MK. Bariatric analysis and reporting outcome system (BAROS). Obes Surg. 1998;8:487-99.

22. Favretti F, Cadiere GB, Segato G, et al. Bariatric analysis and reporting outcome system (BAROS) applied to laparoscopic gastric banding patients. Obes Surg. 1998;8:500-4.

23. Hell E, Miller KA, Moorehead MK, et al. Evaluation of health status and quality of life after bariatric surgery: comparison of standard Roux-en-Y gastric bypass, vertical banded gastroplasty and laparoscopic adjustable silicone gastric banding. Obes Surg. 2000;10:214-9.

24. Victorzon M, Tolonen P. Bariatric Analysis and Reporting Outcome System (BAROS) following laparoscopic adjustable gastric banding in Finland. Obes Surg. 2001;11:740-3.

25. Baltasar A. On BAROS. Bariatric Analysis and Reporting Outcome System. Obes Surg. 1999;9:288.

26. Moorehead MK, Ardelt-Gattinger E, Lechner H, et al. The validation of the Moorehead-Ardelt quality of life questionnaire II. Obes Surg. 2003;13:684-92.

27. The WHOQOL Group. Study protocol for the World Health Organization project to develop a Quality of Life assessment instrument (WHOQOL). Qual Life Res. 1993;2:153-9.

28. The WHOQOL Group. The World Health Organization Quality of Life Assessment (WHOQOL): development and general psychometric properties. Soc Sci Med. 1998;46:1569-85.

29. Anderson RT, Aaronson NK, Bullinger M, et al. A review of the progress towards developing health-related quality-of-life instruments for international clinical studies and outcomes research. Pharmacoeconomics 1996;10:336-55.

30. The WHOQOL Group. Development of the World Health Organization WHOQOL-BREF quality of life assessment. Psychol Med. 1998;28:551-8.

31. Hsiung PC, Fang CT, Chang YY, et al. Comparison of WHOQOL-bREF and SF-36 in patients with HIV infection. Qual Life Res. 2005;14:141-50.

32. Yang SC, Kuo PW, Wang JD, et al. Quality of life and its determinants of hemodialysis patients in Taiwan measured with WHOQOL-BREF(TW). Am J Kidney Dis. 2005;46:635-41. 
33. Hsu C, Wang JD, Hwang JS, et al. Survival-weighted health profile for long-term survivors of acute myelogenous leukemia. Qual Life Res. 2003;12:503-17.

34. Liou HH, Chen RC, Chen CC, et al. Health related quality of life in adult patients with epilepsy compared with a general reference population in Taiwan. Epilepsy Res. 2005;64:151-9.

35. Ware JE Jr, Sherbourne CD. The MOS 36-item short-form health survey (SF-36). I. Conceptual framework and item selection. Med Care. 1992;30:473-83.

36. Duval K, Marceau P, Perusse L, et al. An overview of obesityspecific quality of life questionnaires. Obes Rev. 2006;7:347-60.
37. Montazeri A, Goshtasebi A, Vahdaninia M, et al. The Short Form Health Survey (SF-36): translation and validation study of the Iranian version. Qual Life Res. 2005;14:875-82.

38. Koh ET, Leong KP, Tsou IY, et al. The reliability, validity and sensitivity to change of the Chinese version of SF-36 in oriental patients with rheumatoid arthritis. Rheumatology 2006;45: 1023-8.

39. Gandek B, Ware JE Jr, Aaronson NK, et al. Tests of data quality, scaling assumptions, and reliability of the SF-36 in eleven countries: results from the IQOLA Project. International Quality of Life Assessment. J Clin Epidemiol. 1998;51:1149-58. 